\title{
Chirurgische Gastroenterologie InterdisziplinäR wird zu ViszeralmedizIN
}

\author{
Ernst Klar $^{\mathrm{a}}$ Joachim Mössner ${ }^{\mathrm{b}}$ Ansgar W. Lohse ${ }^{\mathrm{c}}$ \\ a Allgemeine, Thorax-, Gefäß- und Transplantationschirurgie, Klinik und Poliklinik für Chirurgie, Universität Rostock \\ ${ }^{\mathrm{b}}$ Gastroenterologie/Hepatologie, Medizinische Klinik II, Universitätsklinikum Leipzig, \\ ${ }^{\mathrm{C}}$ I. Medizinische Klinik und Poliklinik, Universitätsklinikum Hamburg-Eppendorf, Hamburg, Deutschland
}

Viszeralmedizin ist ein neues Journal mit altem und bewährtem Ursprung. Vor 25 Jahren wurde die Zeitschrift CHIRURGISCHE GASTROENTEROLOGIE MIT INTERDISZIPLINÄREN GESPRÄCHEN von den Kollegen A. Akovbiantz, H. Denk, K.-J. Paquet und C.E. Zöckler gegründet, mit dem Ziel, die fachübergreifende Diskussion von Schwerpunktthemen zu fördern. Dieses Konzept ist bei den Lesern auf besonderes Interesse gestoßen, auch deshalb, weil den Hauptthemen eine interdisziplinäre Diskussion von Brennpunkten durch unabhängige Experten angeschlossen wurde. Diese Grundidee der Zeitschrift wurde jetzt eingeholt durch das Zusammenwachsen von Chirurgie und Gastroenterologie eben im Sinne des neuen Titels unserer Zeitschrift, der Viszeralmedizin. Der gleichnamige Kongress der Deutschen Gesellschaft für Verdauungs- und Stoffwechselkrankheiten und der Deutschen Gesellschaft für Allgemein- und Viszeralchirurgie dokumentiert ganz wesentlich diese Entwicklung der letzten Jahre. Die Zeitschrift VISZERALMEDIZIN wird deshalb eng an diesen Kongress angebunden sein und die exzellenten interdisziplinären Sitzungen in Schriftform wiedergeben. Die gemeinsamen Sitzungen sind State-of-the-Art-Analysen in der Darstellung der jeweils fachspezifischen Aspekte und dienen der gemeinsamen Diskussion zwischen Chirurgen und Gastroenterologen. Das hier vorliegende erste Heft in neuer Konzeption zeigt sicher am Besten das zukünftige Charakteristikum der Zeitschrift: die ausgezeichneten Vorträge ausgesuchter Brennpunktsitzungen werden unter der Regie der Sitzungsvorsitzenden als Übersichtsartikel einer breiten Leserschaft zugänglich gemacht. Die Submukosakarzinome stellen eine exemplarische Schnittstelle zwischen endoskopischer und operativer Therapie dar. Die Optimierung der Konzepte besitzt hohe Kinetik. Die aktuelle Bestandsaufnahme wird akzentuiert durch die Beantwortung von Schlüsselfragen im Interdisziplinären Gespräch. Die ViszeralmedizIn wird auch Originalarbeiten und Kasuistiken integrieren; die Betonung liegt auf «Der besondere Fall».

Das Editorial Board hat sich entsprechend viszeralmedizinischer Schwerpunktbildung und Interdisziplinarität verändert. Das Echo auf unser Konzept ist ausgesprochen positiv, und wir danken den Herausgebern des vorliegenden Themenheftes und der schon konzipierten Ausgaben sowie den Autoren für die bereitwillige und tatkräftige Unterstützung gerade in dieser Initialphase. Im Sinne unserer Patienten soll die VISZERALmedizIN krankheitsorientierte, fachübergreifende Problemlösungen unterstützen und widerspiegeln. Die Herausgeber freuen sich darauf, die Zeitschrift zusammen mit dem Editorial Board weiterzuentwickeln.

\section{KARGER}

Fax +497614520714

Information@Karger.de

www.karger.com (c) 2009 S. Karger GmbH, Freiburg
Prof. Dr. med. Ernst Klar

Allgemeine, Thorax-, Gefäß- und Transplantationschirurgie

Klinik und Poliklinik für Chirurgie, Universität Rostock

Schillingallee 35, 18055 Rostock, Deutschland

Tel. +49 381494 60-00, Fax -02

ernst.klar@med.uni-rostock.de 